\title{
Invisible Energy from KASCADE Data
}

\author{
Jakub Vícha*, Jan Ebr, Petr Trávníček \\ FZU, Institute of Physics of the Czech Academy of Sciences, Prague, Czech Republic \\ E-mail: vicha@fzu.cz
}

\section{Dalibor Nosek, Vladimír Novotný}

Faculty of Mathematics and Physics, Charles University, Prague, Czech Republic

\begin{abstract}
We applied a data-driven method to derive the invisible energy for air showers using the publicly available data of the KASCADE experiment. The universal relation between the invisible energy and the number of muons measured by the shielded KASCADE detectors was adopted. In this way, we determine the invisible energy for the first time from measured data between $\mathrm{PeV}$ and $\mathrm{EeV}$ energies. We also present new energy calibration of the signals induced in KASCADE detectors exploiting the lateral shape parameter and reducing the bias on the estimated shower energy.
\end{abstract}

36th International Cosmic Ray Conference -ICRC2019-

July 24th - August 1st, 2019

Madison, WI, U.S.A.

${ }^{*}$ Speaker. 


\section{Introduction}

Very-high energy cosmic rays $\left(10^{15-18} \mathrm{eV}\right.$, VHECR) are charged particles considered to originate dominantly inside our Galaxy. Their energy spectrum is of a power-law shape $\left(\propto E^{-\gamma}\right)$ with spectral index $\gamma \sim 2.7$ and it exhibits several features: a softening around $3 \mathrm{PeV}$ (knee), a hardening around $20 \mathrm{PeV}$ (ankle-like feature) and another softening around $100 \mathrm{PeV}$ (the second knee) that may indicate acceleration limits of their sources for different primary particles [1,2].

The primary VHECR generate air showers of secondary particles (mainly muons, electrons and photons) that can reach arrays of ground detectors even at the sea level. The ground detection relies on the energy calibration of a signal induced by air shower using Monte Carlo (MC) simulations with models of hadronic interactions (HI models). These models extrapolate properties of particle interactions (cross-sections, multiplicities, elasticities etc.) measured at accelerators with beam energies (up to $13 \mathrm{TeV}$ for proton-proton collisions at LHC in c.m. system) comparable to the energies of the first interactions of cosmic rays with energy $\sim 10^{17} \mathrm{eV}$. However, measurements at accelerators cover lower pseudorapidity regions than that one covered by most of the energy flow of the first interactions of cosmic rays in the atmosphere. The problems of HI models tuned to the LHC data to describe air-shower data of VHECR (especially the muon component of shower) were reported in [3] for KASCADE-Grande. The inconsistency in the description of measured numbers of muons using MC simulations is higher at ultra-high energies (above $1 \mathrm{EeV}$ ), see $[4,5,6]$.

On the other hand, the secondary particles of a VHECR shower produce also faint isotropic fluorescence and collimated Cherenkov light during their propagation towards the Earth surface. This light can be observed with optical telescopes during night measuring the deposited calorimetric energy of the shower $[7,8,9]$. To reconstruct the energy of the primary cosmic ray, this calorimetric energy needs to be further corrected for the energy that is not deposited in the atmosphere. This so-called invisible energy is carried by muons and neutrinos, and is a substantial source of systematic uncertainties in the energy spectrum measured by optical telescopes below $1 \mathrm{EeV}$ (in the case of TALE experiment $20 \%$ [9]).

So far, the invisible energy of VHECR was estimated from MC simulations for given mass composition of VHECR despite the fact that HI models have problems to describe the measured air-shower data and, therefore, also the mass composition of VHECR is burdened by high systematic uncertainties. Moreover, the correctness of the MC description of muon component, that is responsible for a large fraction of the invisible energy, is at least questionable. At ultra-high energies, a data-driven method was applied to the hybrid data of fluorescence and ground detectors of the Pierre Auger Observatory above $10^{18.6} \mathrm{eV}$ and extrapolated down to $10^{17} \mathrm{eV}$ [10], where also a phenomenological justification of the data-driven method can be found. This way, the invisible energy was found even larger than the MC simulations predict for iron primaries.

In this work, we applied a data-driven method to derive the invisible energy from the publicly available VHECR data of the KASCADE experiment. The universal relation between the invisible energy and the number of muons measured by the shielded KASCADE detectors was derived and adopted. In this way, we determine for the first time the invisible energy from measured data between $\mathrm{PeV}$ and $\mathrm{EeV}$ energies. We also derive a new estimation of the total shower energy exploiting the lateral shape parameter and thus reducing the bias on the reconstructed shower energy originating in the fluctuations of the depth of shower maximum. 


\section{KASCADE Data and Simulations}

The KASCADE experiment [11] measured VHECR since 1996 and finished its measurement in 2003. The detected showers were reconstructed using signals in shielded (signals dominantly from muons above $230 \mathrm{MeV}$ ) and unshielded scintillation detectors (signals from charged particles). The collected data of this experiment were released in 2017 together with simulations at the reconstruction level including the detector effects [12].

Both simulated and measured data contain information on the reconstructed number of muons on the ground with energy threshold $230 \mathrm{MeV}\left(N_{\mu}^{\mathrm{Rec}}\right)$, number of electrons $\left(N_{\mathrm{el}}^{\mathrm{Rec}}\right)$, the zenith angle $\left(\Theta_{\operatorname{Rec}}\right)$, the lateral shape parameter $\left(s^{\mathrm{Rec}}\right)$, and the shower energy $\left(E_{\mathrm{Rec}}\right)$. The KASCADE simulations contain additionally to the reconstructed quantities also the information on the true number of muons above $100 \mathrm{MeV}\left(N_{\mu}^{\mathrm{MC}}\right)$, true number of electrons $\left(N_{\mathrm{el}}^{\mathrm{MC}}\right)$, true zenith angle $\left(\Theta_{\mathrm{MC}}\right)$, primary energy $\left(E_{\mathrm{MC}}\right)$ and the type of primary particle initiating the generated shower.

However, the calorimetric and invisible energies are not included in KASCADE simulations. Therefore, we produced an additional library of showers simulated using the CORSIKA code [13] with the same settings as the publicly available KASCADE simulations to obtain the information on the invisible energy $\left(E_{\text {Inv }}\right)$ and to finally relate it with the numbers of generated muons.

\subsection{KASCADE Data}

We used NABOO 2.0 version [12] of released data for runs 4685-7417 containing 252,658,250 reconstructed showers from period 08.05.1998 -20.12.2003 with zenith angle $\Theta_{\operatorname{Rec}}=0^{\circ}-60^{\circ}$, azimuth angle $0^{\circ}-360^{\circ}$, lateral shape parameter $s^{\operatorname{Rec}}=0.1-1.48$, core positions in the square of size $91 \mathrm{~m}$ centred in the middle of the KASCADE experiment with $\log _{10} N_{\mathrm{el}}^{\mathrm{Rec}}$ and $\log _{10} N_{\mu}^{\mathrm{Rec}}$ both higher than 2 . This pre-selection of data guarantees a constant quality of the measured data.

We applied cuts on the reconstructed numbers of particles $\log _{10} N_{\mathrm{el}}^{\mathrm{CUT}}=4.4$ and $\log _{10} N_{\mu}^{\mathrm{CUT}}=$ 4.0. We applied also additional cuts recommended by the KASCADE group to maintain high quality of the reconstructed data [14]: $s_{\text {low }}^{\text {CUT }}=0.6, s_{\text {high }}^{\text {CUT }}=1.3$ to cut finally showers with $\log _{10} N_{\mathrm{el}}^{\text {Rec }}<$ $\log _{10} N_{\mathrm{el}}^{\mathrm{CUT}}, \log _{10} N_{\mu}^{\mathrm{Rec}}<\log _{10} N_{\mu}^{\mathrm{CUT}}$, and $s^{\mathrm{Rec}}<s_{\text {low }}^{\mathrm{CUT}}$ and $s^{\mathrm{Rec}}>s_{\text {high }}^{\mathrm{CUT}}$.

For our analysis, we use only showers with $\Theta_{\operatorname{Rec}} \leq 25^{\circ}$ since the formula for estimation of the shower energy using $\log _{10} N_{\mathrm{el}}^{\mathrm{Rec}}$ and $\log _{10} N_{\mu}^{\mathrm{Rec}}$ was derived for $\Theta_{\mathrm{MC}} \leq 25^{\circ}$ [12]. Finally, $16,302,464$ measured showers were used to calculate the invisible energy from the measured numbers of muons.

\subsection{KASCADE Simulations}

The publicly available simulations (see [15] for more details) comprise air showers generated with the CORSIKA program. The detector responses of particles reaching the ground level of the KASCADE experiment are simulated with the program CRES. These showers are finally reconstructed with the program KRETA. The energy of primary particles followed an energy spectrum with spectral index $\gamma_{\mathrm{MC}}=2$ for energies from $10^{14} \mathrm{eV}$ to $10^{18} \mathrm{eV}$, with high-energy extension to $3.16 \cdot 10^{18} \mathrm{eV}$. For the purpose of our analysis, we reweighted the simulated showers to correspond to the energy spectrum of measured data with a spectral index $\sim 2.7$. The generated zenith angle covered range between $0^{\circ}$ and $42^{\circ}$, and the azimuth angle was uniformly distributed between $0^{\circ}$ and $360^{\circ}$. The transition energy between the low energy model FLUKA [16] and the HI models 
was set to $200 \mathrm{GeV}$. The particle densities were sampled at $110 \mathrm{~m}$ a.s.l. which is the altitude of the KASCADE experiment with $X_{0}=1020 \mathrm{~g} / \mathrm{cm}^{2}$ of vertical atmospheric depth. The geomagnetic field in simulations reflected the geomagnetic field of the KASCADE location. In our analysis, we use showers simulated with EPOS-LHC [17], QGSJet II-04 [18] and Sibyll 2.3 [19] for p, He, C and Fe primaries. The energy cut-off for electrons, photons and neutral pions was set to $3 \mathrm{MeV}$ and for muons and hadrons to $100 \mathrm{MeV}$ [15].

The same cuts as the cuts applied to the set of measured data were applied in these simulations, except $s_{\text {low }}^{\mathrm{CUT}}=0.0$ as recommended by the KCDC group [15]. We checked that these cuts keep full reconstruction efficiency of all primary particles above the shower energy $10^{15.3} \mathrm{eV}$. The trigger of the KASCADE array was fully efficient above $\sim 1 \mathrm{PeV}$ [14].

Finally, we obtained about 30,000 selected showers of 500,000 showers at disposal for given HI model and primary particle within $\Theta_{\mathrm{MC}}=25^{\circ}$.

\subsection{Additional CORSIKA Simulations}

We produced a library of simulated showers using CORSIKA 7.64. It contains 60,000 showers generated with the same three HI models as in the case of the KASCADE simulations. The lowenergy model FLUKA 2011.2x was adopted for four primary particles: $\mathrm{p}, \mathrm{He}, \mathrm{N}$ and Fe. Ten fixed values of the zenith angle were distributed uniformly in $\cos ^{2} \Theta_{M C}\left(\Theta_{M C}=0^{\circ}, 12.3^{\circ}, 17.6^{\circ}, 21.8^{\circ}\right.$, $25.4^{\circ}, 28.6^{\circ}, 31.7^{\circ}, 34.5^{\circ}, 37.3^{\circ}$ and $\left.40^{\circ}\right)$ for each of five fixed primary energies $\left(E_{\mathrm{MC}}=10^{15} \mathrm{eV}\right.$, $3.2 \cdot 10^{15} \mathrm{eV}, 10^{16} \mathrm{eV}, 3.2 \cdot 10^{16.5} \mathrm{eV}$ and $10^{17} \mathrm{eV}$ ). For each HI model, each primary particle, each energy and each zenith angle, 100 showers were generated. The settings of CORSIKA simulations were adjusted according to the settings mentioned in Sec. 2.2.

The calorimetric energy was calculated for each simulated shower $\left(E_{\mathrm{Cal}}^{\mathrm{MC}}\right)$ as the sum of energy deposited by charged particles at each depth of shower until the ground level. A correction for a part of the calorimetric energy below the ground was accounted for. The invisible energy for individual shower was then obtained as $E_{\mathrm{Inv}}^{\mathrm{MC}}=E_{\mathrm{MC}}-E_{\mathrm{Cal}}^{\mathrm{MC}}$.

The number of muons $\left(N_{\mu}^{\mathrm{MC}}\right)$ was obtained as a sum of all muons (above $100 \mathrm{MeV}$ ) reaching the ground level as in the case of $N_{\mu}^{\mathrm{MC}}$ in KASCADE simulations. The relative difference of $\left\langle N_{\mu}^{\mathrm{MC}}\right\rangle$ between the KASCADE simulations and the additional CORSIKA simulations was found to be within $0.5 \%$.

\section{Invisible Energy}

The additional CORSIKA simulations were used to calibrate the invisible energy $E_{\text {Inv }}$ with $N_{\mu}^{\mathrm{MC}}$ for each of ten fixed zenith angles (see one example in Fig. 1a) according to $E_{\mathrm{Inv}}\left(N_{\mu}^{\mathrm{MC}}\right)=$ $C \cdot\left(N_{\mu}^{\mathrm{MC}}\right)^{\delta}$. The showers of three HI models, four primaries and five energy bins were fitted with the least square method (black line). The obtained parameters $C$ and $\delta$ are depicted in Fig. $1 \mathrm{~b}$ for different $\Theta_{\mathrm{MC}}$. The zenith-angle dependences of parameters $C$ and $\delta$ were fitted with quadratic functions of $\cos ^{2}\left(\Theta_{\mathrm{MC}}\right)$ using the least square method (black lines). These zenith-angle dependences come mainly from the attenuation of $N_{\mu}^{\mathrm{MC}}$. The difference of $E_{\mathrm{Inv}}^{\mathrm{MC}}$ between $\Theta_{\mathrm{MC}}=0^{\circ}$ and $\Theta_{\mathrm{MC}}=25^{\circ}$ was found to be within $\sim 2 \%$. The average values of the residuals $\left(\Delta E_{\text {Inv }} / E_{\text {Inv }}\right)$ of fitted $E_{\text {Inv }}$ per primary and $\mathrm{HI}$ model are within $20 \%$ for all $\Theta_{\mathrm{MC}} \leq 25^{\circ}$. 


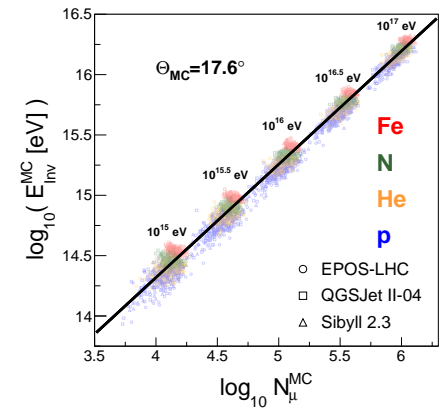

(a) $E_{\text {Inv }}^{\mathrm{MC}}$ vs. $N_{\mu}^{\mathrm{MC}}$ example
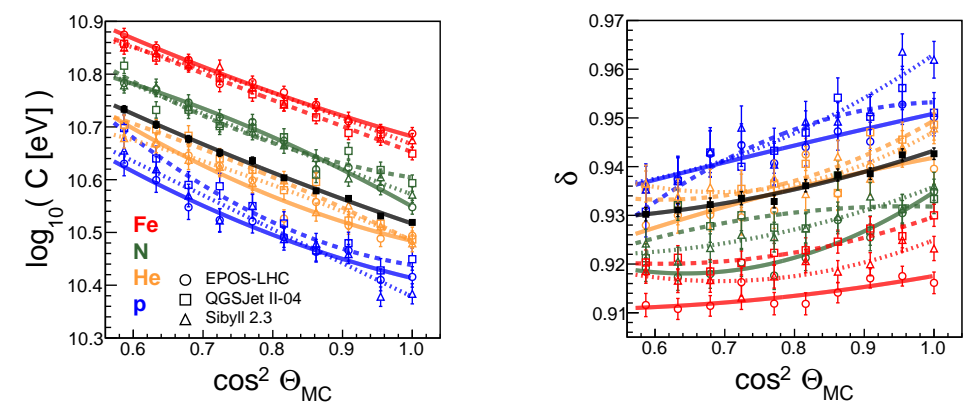

(b) Zenith evolution of $E_{\text {Inv }}$ calibration coefficients

Figure 1: Left panel (a): Calibration of the invisible energy with the numbers of muons for $\Theta_{\mathrm{MC}}=$ $17.6^{\circ}$. The evolution of fitted parameters with $\Theta_{M C}$ is illustrated in the middle and right panels (b). Fits of showers produced with three HI models and four primaries are depicted by black lines.

\section{Calorimetric Energy}

An estimate of the $E_{\text {Inv }}$ applicable to the optical measurements is usually provided in the form of the fraction of the total shower energy $\left(E_{\mathrm{Tot}}\right)$ as $E_{\mathrm{Inv}} / E_{\mathrm{Tot}}=E_{\mathrm{Inv}} / E_{\mathrm{Tot}}\left(\log _{10}\left(E_{\mathrm{Cal}}[\mathrm{eV}]\right)\right.$. The calorimetric energy, $E_{\mathrm{Cal}}=E_{\mathrm{Tot}}-E_{\mathrm{Inv}}$, (or the total shower energy) is therefore another quantity that is needed to provide the invisible energy in a suitable form for optical measurements.

We use two different estimations of $E_{\mathrm{Cal}}$ in the following. Firstly, $E_{\mathrm{Cal}}$ is estimated from the total energy reconstructed by the KASCADE Collaboration. Secondly, we introduce a calibration of $E_{\mathrm{Cal}}$ with the number of charged particles for a given value of the lateral shape parameter $s_{\mathrm{Rec}}$.

\section{1 $\mathrm{E}_{\mathrm{Cal}}$ from the KASCADE Reconstructed Energy}

In the case of the KASCADE experiment, the shower energy $\left(E_{\mathrm{Rec}}\right)$ is estimated from the measured number of muons and electrons on the ground [12], accounting for their attenuations. It is based on the comparison with signals of showers generated for HI model QGSJet II-02 and Fluka 2002_4. Such energy calibration is substantially biased wrt. the mass composition and HI models (see left panel of Fig. 4). To avoid these shortcomings, we chose a combination of the four primaries ( $\mathrm{p}, \mathrm{He}, \mathrm{C}$ and $\mathrm{Fe}$ ) developing with the shower energy according to the Global Spline Fit (GSF) model [2] for primary fractions of p, He, CNO group and Fe group, respectively, and corrected $E_{\mathrm{Rec}}$ for average mass composition bias $\left(E_{\mathrm{Rec}}^{*}\right)$. We applied a rescaling of the energy scale in GSF by 0.88 to account for the energy rescaling applied in [2] for KASCADE-Grande. We consider the same energy scale for the KASCADE experiment (energy calibrations using the same $\mathrm{MC}$ simulations). The calorimetric energy is finally obtained as $E_{\mathrm{Cal}}=E_{\mathrm{Rec}}^{*}-E_{\mathrm{Inv}}$.

\section{2 $\mathrm{E}_{\mathrm{Cal}}$ from the Number of Charged Particles and the Lateral Shape Parameter}

The relationships between the invisible energy and the number of muons derived for given HI model and primary (lines of different colors in Fig. 1b) show average residuals within 3\%. We used these relationships to derive the calorimetric energy per shower $\left(E_{\mathrm{Cal}}^{\mathrm{MC}}\right)$ of given $\mathrm{HI}$ model and primary of the KASCADE simulations as $E_{\mathrm{Cal}}^{\mathrm{MC}}=E_{\mathrm{MC}}-E_{\mathrm{Inv}}\left(N_{\mu}^{\mathrm{MC}}\right)$. The lateral shape parameter 
$s_{\text {Rec }}$ is the slope of the lateral distribution function of the number of electrons. This parameter is related to the mass distance of the depth of shower maximum to the ground. We assumed that for a given $s_{\text {Rec }}$, the number of charged particles on the ground transformed to the zenithangle of $0^{\circ}\left(N_{\mathrm{ch}}^{\mathrm{MC}}\left(0^{\circ}\right)=N_{\mathrm{el}}^{\mathrm{MC}}\left(0^{\circ}\right)+N_{\mu}^{\mathrm{MC}}\left(0^{\circ}\right)\right)$ is proportional to the calorimetric energy of shower (see Fig. 2a). We fitted showers of all HI models and primaries for different bins in $s_{\text {Rec }}$ with $E_{\text {Cal }}\left(N_{\mathrm{ch}}^{\mathrm{MC}}\left(0^{\circ}\right)\right)=D \cdot\left(N_{\mathrm{ch}}^{\mathrm{MC}}\left(0^{\circ}\right)\right)^{\omega}$ to obtain the parametrization of the calibration coefficients with $s_{\text {Rec }}$ (see Fig. 2b). Finally, we applied a correction on $E_{\text {Cal }}$ due to the evolution of $s_{\text {Rec }}$ with energy that is universal for HI models and primaries.

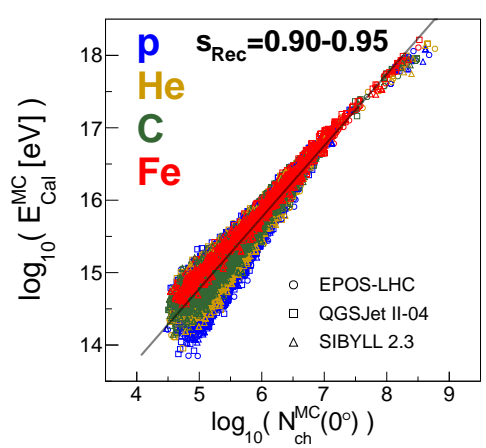

(a) $E_{\text {Cal }}^{\mathrm{MC}}$ vs. $N_{\mathrm{ch}}^{\mathrm{MC}}$ example

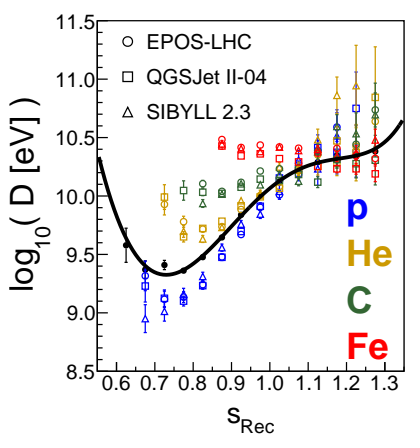

(b) Evolution of $E_{\mathrm{Cal}}$ calibration coefficients with age parameter

Figure 2: Left panel (a): Calibration of calorimetric energy with the numbers of charged particles for one bin of the shower age parameter $s_{\text {Rec }}$. Right panels (b): Evolution of coefficients of the $E_{\text {Cal }}$ calibration with $s_{\text {Rec }}$. Fits to all primaries and HI models are depicted by black lines.

\section{Fraction of Invisible Energy from KASCADE Data}

In Fig. 3, the mean fractions of the invisible energy are plotted as a function of the logarithm of the calorimetric energy. We show the three cases when the calorimetric energy was derived from the KASCADE reconstructed energy which was corrected for the average energy bias for each HI model $\left(E_{\mathrm{Tot}}=E_{\mathrm{Rec}}^{*}\right)$. The full crosses depict the mean fractions of $E_{\mathrm{Inv}}$ for $E_{\mathrm{Cal}}$ being estimated from $N_{\mathrm{ch}}\left(0^{\circ}\right)$ and $s_{\mathrm{Rec}}\left(E_{\mathrm{Tot}}=E_{\mathrm{Cal}}+E_{\mathrm{Inv}}\right)$. The systematic uncertainties (gray band and brackets) are dominated by remaining dependencies on HI model and mass composition (calibrations of $E_{\text {Inv }}$, $E_{\mathrm{Cal}}$ and bias estimation of $\left.E_{\mathrm{Rec}}\right)$. The difference between the fractions of the invisible energy for the two energy calibrations is now unclear and needs further study.

For comparison, we plot MC predictions for protons (blue) and iron nuclei (red) obtained with CONEX $6.40[20,21]$ simulations for zenith angles within $25^{\circ}$ and energies from $10^{15} \mathrm{eV}$ up to $10^{20} \mathrm{eV}$. We observe three regions of $\log _{10}\left(E_{\mathrm{Cal}}[\mathrm{eV}]\right)$ where a sudden change occurs in all cases for KASCADE data (breaks at $\log _{10}\left(E_{\mathrm{Cal}}[\mathrm{eV}]\right)=\sim 15.8, \sim 16.6$ and $\sim 17.2$ ). The last break is less significant due to the limited statistics. We did not find any dependence of the results $(<2 \%)$ on $\Theta_{\text {Rec }}$ within $25^{\circ}$. These preliminary results are below the extrapolation of AUGER measurements [10] in the common range of $\log _{10}\left(E_{\mathrm{Cal}}[\mathrm{eV}]\right)=17.0-17.4$. 


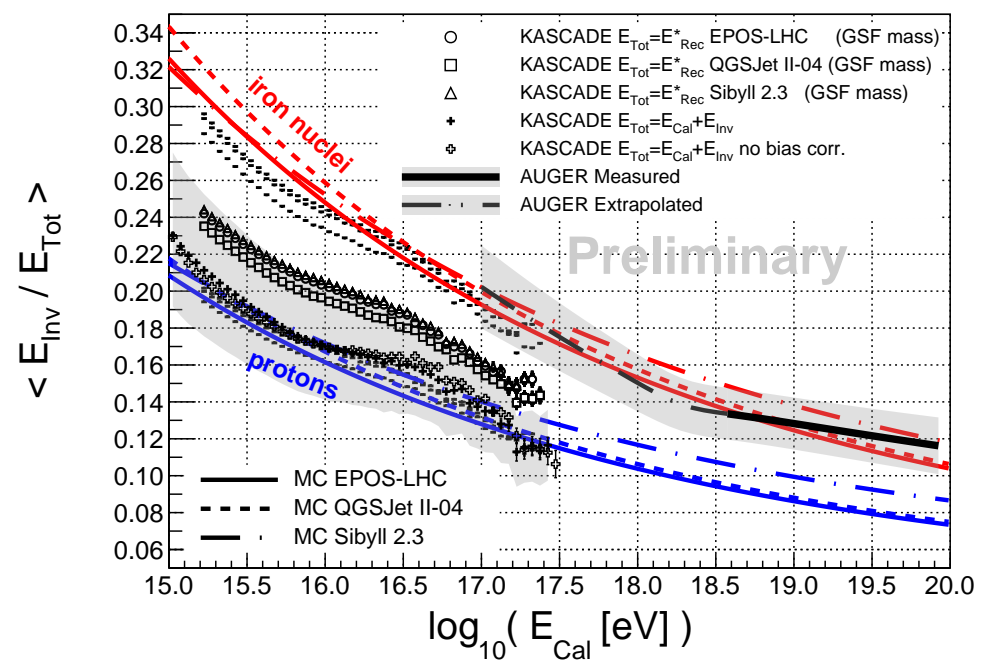

Figure 3: Invisible energy as a fraction of shower energy depending on calorimetric energy.

\section{New Energy Calibration}

The estimation of the shower energy $\left(E_{\mathrm{Rec}}\right)$ as a sum of the invisible (from $N_{\mu}^{\mathrm{Rec}}, \Theta_{\mathrm{Rec}}$ ) and calorimetric energy (from $N_{\mu}^{\mathrm{Rec}}, N_{\mathrm{el}}^{\mathrm{Rec}}, \Theta_{\mathrm{Rec}}$ and $s_{\mathrm{Rec}}$ ) has much smaller bias (within $\sim \pm 20 \%$ ) with current $\mathrm{HI}$ models than the bias of estimation of $E_{\operatorname{Rec}}$ by KASCADE (from $\sim-20 \%$ to $\sim+70 \%$ ) using a quadratic function of $\log _{10} N_{\mu}^{\text {Rec }}$ and $\log _{10} N_{\mathrm{el}}^{\mathrm{Rec}}$, both corrected for $\Theta_{\mathrm{Rec}}$, see Fig. 4.

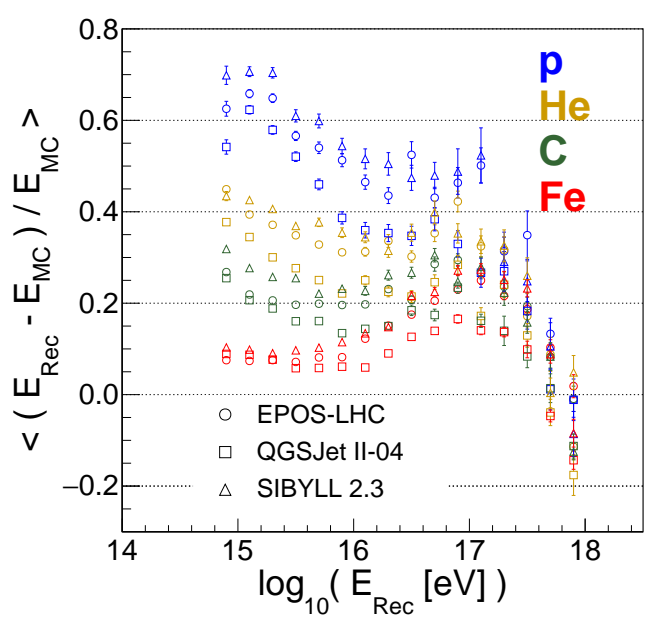

(a) Bias of KASCADE Energy Estimation

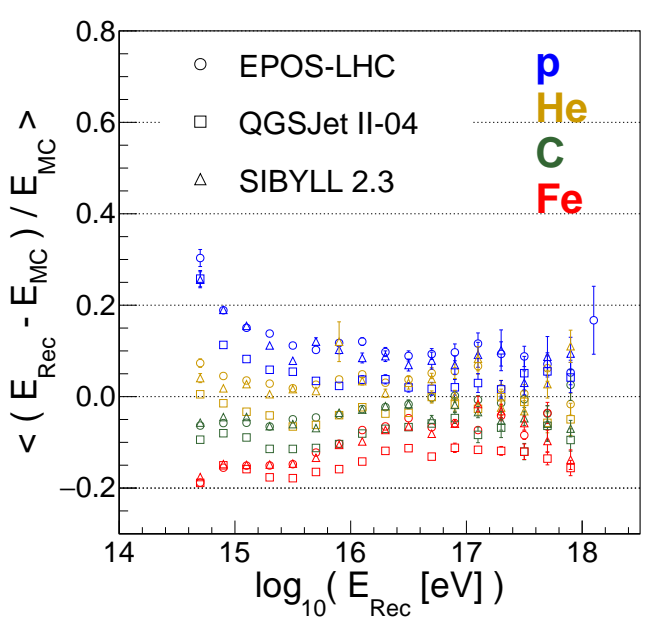

(b) Bias of New Energy Estimation

Figure 4: Biases of the shower energy estimated using KASCADE formula (left (a)) and using calibrations of $E_{\mathrm{Inv}}\left(N_{\mu}^{\mathrm{Rec}}\right)$ and $E_{\mathrm{Cal}}\left(N_{\mathrm{ch}}\left(0^{\circ}\right), s_{\mathrm{Rec}}\right)$ (right (b)).

\section{Acknowledgements}

This work is funded by Ministry of Education, Youth and Sports of the Czech Republic under 
the projects LTT18004, LM2015038 and CZ.02.1.01/0.0/0.0/16_013/0001402.

\section{References}

[1] W.D. Apel et al., KASCADE-Grande measurements of energy spectra for elemental groups of cosmic rays, Astropart. Phys. 47 (2013) 54.

[2] H. Dembinski et al., Data-driven model of the cosmic-ray flux and mass composition from $10 \mathrm{GeV}$ to $10^{11} \mathrm{GeV}$, proceedings of the ICRC 2017, PoS(ICRC 2017)533.

[3] W.D. Apel et al., Probing the evolution of the EAS muon content in the atmosphere with KASCADE-Grande, Astropart. Phys. 95 (2017) 25.

[4] A. Aab et al., Testing Hadronic Interactions at Ultrahigh Energies with Air Showers Measured by the Pierre Auger Observatory, Phys. Rev. Lett. 117 (2016) 192001.

[5] A. Aab et al., Muons in air showers at the Pierre Auger Observatory: Mean number in highly inclined events, Phys. Rev. D 91 (2015) 032003.

[6] A. Aab et al., Erratum: Muons in air showers at the Pierre Auger Observatory: Measurement of atmospheric production depth, Phys. Rev. D 90 (2015) 019903.

[7] J. Abraham et al., The fluorescence detector of the Pierre Auger Observatory, NIM A 620 (2010) 227.

[8] R. U. Abbasi et al., Study of Ultra-High Energy Cosmic Ray composition using Telescope Array's Middle Drum detector and surface array in hybrid mode, Astropart. Phys. 64 (2015) 49.

[9] R. U. Abbasi et al., The Cosmic Ray Energy Spectrum between 2 PeV and 2 EeV Observed with the TALE Detector in Monocular Mode, AAS 865 (2018) 74.

[10] A. Aab et al., Data-driven estimation of the invisible energy of cosmic ray showers with the Pierre Auger Observatory, submitted to Phys. Rev. D (2019), arXiv:1901.08040 [astro-ph.IM].

[11] T. Antoni et al., The cosmic-ray experiment KASCADE, NIM A 513 (2003) 490.

[12] A. Haungs et al., The KASCADE Cosmic-ray Data Centre KCDC: granting open access to astroparticle physics research data, EPJ C 78 (2018) 741.

[13] D. Heck et al., Upgrade of the Monte Carlo code CORSIKA to simulate extensive air showers with energies $>10^{20} \mathrm{eV}$, Report FZKA Forschungszentrum Karlsruhe 6019 (1998).

[14] KIT, Karlsruhe Institute of Technology, KCDC User Manual, V.17 from 2018-07-13.

[15] KIT, Karlsruhe Institute of Technology, KCDC Simulation Manual, V.01.1 from 2017-10-30.

[16] A. Ferrari et al., FLUKA: A multi-particle transport code (Program version 2005), Report CERN-2005-010 (2005).

[17] T. Pierog et al., EPOS LHC: Test of collective hadronization with data measured at the CERN Large Hadron Collider, Phys. Rev. C 92 (2015) 034906.

[18] S. Ostapchenko, Monte Carlo treatment of hadronic interactions in enhanced Pomeron scheme: QGSJET-II model, Phys. Rev. D 83 (2011) 014018.

[19] F. Riehn et al., A new version of the event generator Sibyll, proceedings of the ICRC 2015, PoS(ICRC2015)558.

[20] T. Bergmann et al., One-dimensional Hybrid Approach to Extensive Air Shower Simulation, Astropart. Phys. 151 (2006) 159.

[21] T. Pierog et al., First results of fast one-dimensional hybrid simulation of EAS using CONEX, Nucl. Phys. Proc. Suppl. 26 (2007) 420. 